\title{
Correction to: The novel cutoff points for the FIB4 index categorized by age increase the diagnostic accuracy in NAFLD: a multi-center study
}

\author{
Hiroshi Ishiba ${ }^{1,2} \cdot$ Yoshio Sumida $^{3}$ Saiyu Tanaka ${ }^{4}$ Masato Yoneda $^{5} \cdot$ \\ Hideyuki Hyogo $^{6} \cdot$ Masafumi Ono $^{7} \cdot$ Hideki Fujii $^{8} \cdot$ Yuichiro Eguchi $^{9}$ • \\ Yasuaki Suzuki $^{10}$ - Masashi Yoneda ${ }^{3}$ - Hirokazu Takahashi ${ }^{9}$ 'Takashi Nakahara ${ }^{11}$. \\ Yuya Seko $^{2} \cdot$ Kojiro Mori $^{4} \cdot$ Kazuyuki Kanemasa $^{4} \cdot$ Keiji Shimada $^{12}$. \\ Sunsuke Imai ${ }^{12}$ - Kento Imajo ${ }^{5}$ Takumi Kawaguchi ${ }^{13}$ - Atsushi Nakajima ${ }^{5}$. \\ Kazuaki Chayama ${ }^{11}$. Toshiji Saibara ${ }^{7}$ - Toshihide Shima ${ }^{14}$. Kazuma Fujimoto ${ }^{9}$. \\ Takeshi Okanoue ${ }^{14} \cdot$ Yoshito Itoh $^{2}$ for the Japan Study Group of Non-Alcoholic Fatty \\ Liver Disease (JSG-NAFLD)
}

Published online: 25 May 2018

(C) Japanese Society of Gastroenterology 2018

\section{Correction to: J Gastroenterol}

$$
\text { https://doi.org/10.1007/s00535-018-1474-y }
$$

The coauthor Masashi Yoneda's affiliation has been incorrectly published in the original publication of the article. The correct affiliation is provided in this correction.

The original article can be found online at https://doi.org/10.1007/ s00535-018-1474-y.

Yoshio Sumida sumida19701106@yahoo.co.jp

1 Department of Gastroenterology, North Medical Center of Kyoto Prefectural University of Medicine, Yosanocho, Japan

2 Department of Gastroenterology and Hepatology, Kyoto Prefectural University of Medicine, Kyoto, Japan

3 Division of Hepatology and Pancreatology, Department of Internal Medicine, Aichi Medical University, Nagakute, Japan

4 Center for Digestive and Liver Diseases, Nara City Hospital, Nara, Japan

5 Division of Gastroenterology, Yokohama City University Graduate School of Medicine, Yokohama, Japan

6 Department of Gastroenterology, JA Hiroshima Kouseiren General Hospital, Hiroshima, Japan
7 Department of Gastroenterology and Hepatology, Kochi Medical School, Kochi, Japan

8 Department of Hepatology, Graduate School of Medicine, Osaka City University, Osaka, Japan

9 Department of Internal Medicine, Saga Medical School, Saga University, Saga, Japan

10 Department of Gastroenterology, Nayoro City General Hospital, Nayoro, Japan

11 Department of Gastroenterology and Metabolism, Applied Life Sciences, Institute of Biomedical and Health Sciences, Hiroshima University, Hiroshima, Japan

12 Department of Pathology, Nara City Hospital, Nara, Japan

13 Division of Gastroenterology, Department of Medicine, Kurume University School of Medicine, Kurume, Japan

14 Hepatology Center, Saiseikai Suita Hospital, Suita, Japan 